\title{
Oncogenic Src Requires A Wild-Type Counterpart To Regulate Invadopodia Maturation
}

L. C. Kelley

A. G. Ammer

K. E. Hayes

K.H.Martin

K. Machida

See next page for additional authors

Follow this and additional works at: https://researchrepository.wvu.edu/faculty_publications

\section{Digital Commons Citation}

Kelley, L. C.; Ammer, A. G.; Hayes, K. E.; Martin, K. H.; Machida, K.; Jia, L.; Mayer, B. J.; and Weed, S. A., "Oncogenic Src Requires A Wild-Type Counterpart To Regulate Invadopodia Maturation" (2010). Faculty Scholarship. 626.

https://researchrepository.wvu.edu/faculty_publications/626 
Authors

L. C. Kelley, A. G. Ammer, K. E. Hayes, K. H. Martin, K. Machida, L. Jia, B. J. Mayer, and S. A. Weed 


\title{
Oncogenic Src requires a wild-type counterpart to regulate invadopodia maturation
}

\author{
Laura C. Kelley ${ }^{1}$, Amanda Gatesman Ammer ${ }^{1}$, Karen E. Hayes ${ }^{1}$, Karen H. Martin ${ }^{1}$, Kazuya Machida ${ }^{2}$, Lin Jia ${ }^{2}$, \\ Bruce J. Mayer ${ }^{2}$ and Scott A. Weed ${ }^{1, *}$ \\ ${ }^{1}$ Department of Neurobiology and Anatomy, Program in Cancer Cell Biology, Mary Babb Randolph Cancer Center, West Virginia University, \\ Morgantown, West Virginia, 26506-9300, USA \\ ${ }^{2}$ Raymond and Beverly Sackler Laboratory of Genetics and Molecular Medicine, Department of Genetics and Developmental Biology, \\ University of Connecticut Health Center, Farmington, CT 06030, USA \\ *Author for correspondence (sweed@hsc.wvu.edu)
}

Accepted 27 July 2010

Journal of Cell Science 123, 3923-3932

( 2010. Published by The Company of Biologists Ltd

doi:10.1242/jcs.075200

\section{Summary}

The proto-oncogene Src tyrosine kinase ( $\mathrm{Src}$ ) is overexpressed in human cancers and is currently a target of anti-invasive therapies. Activation of Src is an essential catalyst of invadopodia production. Invadopodia are cellular structures that mediate extracellular matrix (ECM) proteolysis, allowing invasive cell types to breach confining tissue barriers. Invadopodia assembly and maturation is a multistep process, first requiring the targeting of actin-associated proteins to form pre-invadopodia, which subsequently mature by recruitment and activation of matrix metalloproteases (MMPs) that facilitate ECM degradation. We demonstrate that active, oncogenic Src alleles require the presence of a wild-type counterpart to induce ECM degradation at invadopodia sites. In addition, we identify the phosphorylation of the invadopodia regulatory protein cortactin as an important mediator of invadopodia maturation downstream of wild-type Src. Distinct phosphotyrosine-based protein-binding profiles in cells forming pre-invadopodia and mature invadopodia were identified by SH2-domain array analysis. These results indicate that although elevated Src kinase activity is required to target actin-associated proteins to pre-invadopodia, regulated Src activity is required for invadopodia maturation and matrix degradation activity. Our findings describe a previously unappreciated role for proto-oncogenic Src in enabling the invasive activity of constitutively active Src alleles.

Key words: Head and Neck cancer, Src, Invadopodia, Cortactin

\section{Introduction}

Src is the first described proto-oncogene and a current target for anti-invasive compounds in clinical trials (Brunton and Frame, 2008; Yeatman, 2004). Src participates in a vast array of cellular functions that include the regulation of cell proliferation, adhesion, migration and invasion (Guarino, 2010; Thomas and Brugge, 1997). In normal cells, Src activity is tightly controlled through intramolecular regulation, subcellular localization and protein expression levels. Elevated or aberrant Src activity is a potent mediator of cell transformation and tumor progression, and is associated with the majority of human cancers including head and neck squamous cell carcinoma (HNSCC) (Summy and Gallick, 2003).

One of the most evident phenotypes of Src-transformed cells is the formation of actin-rich ventral membrane protrusive structures that actively degrade ECM (Chen, 1989). These structures, termed invadopodia, are made by metastatic cancer cells and Srctransformed fibroblasts (Linder, 2009). Invadopodia spontaneously form in tumor cells directly cultured from patient samples and are hypothesized to facilitate breaching of basement membranes during metastasis (Clark et al., 2007; Yamaguchi and Condeelis, 2007). Src activity is absolutely necessary for invadopodia formation and function, and the level of tyrosine phosphorylation at invadopodia positively correlates with the degree of ECM degradation (Bowden et al., 2006; Spinardi et al., 2004). The molecular components that make up invadopodia include proteins that facilitate actin assembly, membrane trafficking and focal degradation. Src substrates participate in all of these functions and include the proteins cortactin
(Bowden et al., 1999), N-WASp (Yamaguchi et al., 2005), dynamin2 (Baldassarre et al., 2003), AMAP1 (Onodera et al., 2005), paxillin (Bowden et al., 1999), p130Cas (Brabek et al., 2004), Tsk5 (Seals et al., 2005), p190RhoGAP (Nakahara et al., 1998), AFAP110 (Gatesman et al., 2004) and caveolin (Yamaguchi et al., 2009). Several studies have evaluated Src activity in invadopodia formation through the ectopic expression of constitutively active Src alleles (Artym et al., 2006; Oser et al., 2009; Stylli et al., 2009). However, these activating Src mutants are rarely found in human tumors, which instead typically contain increased levels of wild-type (WT) Src expression and/or aberrant WT Src activity due to hyperactivation of upstream pathways (Yeatman, 2004). The role of WT Src in invadopodia formation and function is unknown.

Invadopodia assembly has been proposed to involve several stages that regulate the progression from pre-invadopodia (nondegradative) complexes to functional, mature invadopodia containing active MMPs that degrade ECM (Artym et al., 2006; Oser et al., 2009). In current models of invadopodia formation, filamentous (F)-actin and the actin-associated protein cortactin are recruited to sites of matrix adhesion, resulting in pre-invadopodia complexes (Artym et al., 2006). The membrane-bound matrix metalloproteinase MT1-MMP (MMP14) is subsequently recruited to these sites, allowing matrix degradation and invadopodia maturation. Cortactin is an actin-binding protein that is phosphorylated by Src kinase (Head et al., 2003), and is a core invadopodia component. Knockdown of cortactin expression results in decreased invadopodia formation (Artym et al., 2006; Webb et 
al., 2007) and MMP secretion (Clark and Weaver, 2008; Clark et al., 2007), whereas phosphorylation of cortactin is important for regulation of matrix degradation at invadopodia (Ayala et al., 2008).

Recent work on discerning the molecular mechanism regulating actin polymerization before MMP recruitment has identified dynamic regulation of cortactin phosphorylation or dephophorylation downstream of Src to be central to this process (Oser et al., 2009). In this model, cortactin sequesters the actinsevering protein cofilin within pre-invadopodia. Tyrosine phosphorylation of cortactin releases cofilin, which in turn accelerates actin polymerization by severing existing invadopodial actin filaments. In addition, tyrosine phosphorylation of cortactin creates docking sites for the adaptor protein Nck1, which binds and activates the Arp $2 / 3$ activator N-WASp. The combined effect of cofilin activation and N-WASp-mediated Arp2/3 activity serves to increase actin polymerization as pre-invadopodia mature and obtain the ability to degrade ECM. Dephosphorylation of cortactin is proposed to stabilize maturing invadopodia by downregulating actin polymerization through liberation of the Nck1-N-WASp complex, coupled with rebinding of inactive cofilin. Although these data implicate cycles of cortactin phosphorylation and dephosphorylation as crucial in invadopodia maturation, it is not known how the phosphorylation of cortactin and other invadopodia maturation-associated proteins is spatially and temporally orchestrated through upstream kinase-based signaling to drive invadopodia maturation. In this study, we have determined that the presence of endogenous, regulated WT c-Src is required for the maturation of pre-invadopodia complexes induced by oncogenic Src activity into degradative invadopodia. In addition, we show that cortactin phosphorylation downstream of WT c-Src is an important mediator of the maturation process.

\section{Results \\ Elevated Src activity regulates invadopodia formation in HNSCC cell lines}

The introduction of constitutively active viral Src (v-Src) or constitutively active cellular Src (Src527F) has been examined in invadopodia formation in cancer cell lines (Artym et al., 2006; Buschman et al., 2009; Oikawa et al., 2008; Stylli et al., 2009), but the role of endogenous c-Src in invadopodia function is unclear. We analyzed a panel of HNSCC lines for endogenous c-Src activity and total c-Src protein levels (Fig. 1A), as well as the ability of these lines to form spontaneous invadopodia on FITC-gelatin matrix (Fig. 1B; supplementary material Fig. S1A). Two (UMSCC1 and OSC19) out of the six tested lines generate invadopodia that were identified by the colocalization of actin and cortactin-rich yellow aggregates in merged images (Fig. 1B, white arrows) coinciding with areas of focal gelatin degradation (black arrows). UMSCC1 and OSC19 cells had substantially elevated c-Src expression and c-Src activity compared with the UMSCC2, 1483 and MSK921 cell lines that do not make invadopodia (Fig. 1A). The FADU cells had elevated c-Src activity, but failed to generate spontaneous invadopodia, suggesting that elevated Src expression alone is not sufficient to drive invadopodia biogenesis in this line. However, invadopodia are formed and matrix degradation occurs in all the HNSCC cell lines that do not form spontaneous invadopodia (UMSCC2, 1483, FADU and MSK921) following exogenous Src527F expression (Fig. 1B; supplementary material Fig. S1B). These data suggest a cell-linespecific threshold of Src activity (highest in FADU cells) that must be reached to support invadopodia formation and matrix degradation. These results are consistent with the idea that elevated Src activity drives invadopodia biogenesis, and are in agreement with previous work with small-molecule Src inhibitors in HNSCC cell lines, which found a dose-dependent decrease of invadopodia formation and matrix degradation (Ammer et al., 2009). Similar results have also been shown for breast cancer cells (Pichot et al., 2009).

\section{Endogenous Src expression is required for efficient invadopodia-based matrix degradation in HNSCC cells expressing constitutively active Src}

To test the effect of depletion of endogenous c-Src on HNSCC invadopodia formation and function, c-Src expression was knocked down in UMSCC1 cells by RNA interference ( $\mathrm{SrcSi}$ ) (Fig. 2A) and assayed for invadopodia formation and gelatin degradation (Fig. 2B). Endogenous c-Src was depleted by 58\% at 2 days, and by $70 \%$ at 3 days after transfection. Src-knockdown cells had no statistically significant difference in the number of cells with invadopodia or the number of invadopodia per cell (Fig. 2C, top and middle panels). However, SrcSi cells exhibited a $57 \%$ decrease
A
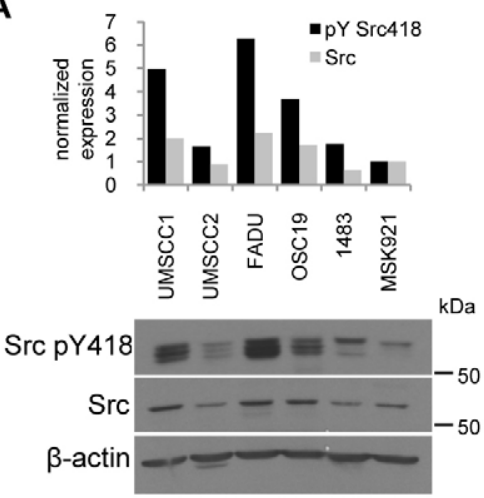

B

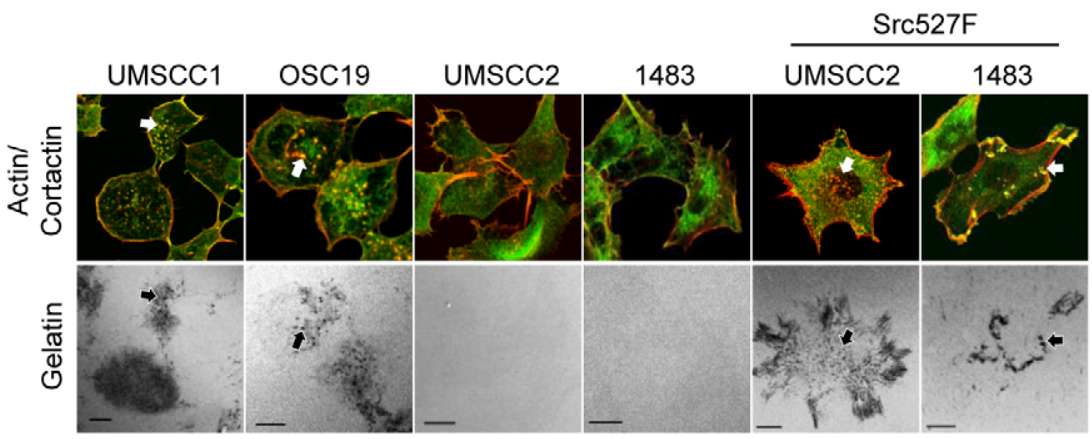

Fig. 1. Src activity regulates invadopodia formation in HNSCC lines. (A) Protein levels of active c-Src (Src-pY $\left.{ }^{418}\right)$, total c-Src (Src) and $\beta$-actin (loading control) in HNSCC lines. For quantification of active Src and total Src, expression levels were normalized to MSK921 cells, a line with low Src activity that does not form invadopodia. The multiple bands in the Src-pY ${ }^{418}$ panel presumably represent additional Src Family kinases; see Fig. 3A. (B) HNSCC cell lines with or without Src-527F were incubated on FITC-gelatin (pseudocolored white) coverslips for 12 hours and labeled with TRITC-phalloidin (red) and anti-cortactin (green). Invadopodia are identified by the yellow aggregates in the merged images of actin and cortactin (white arrows) that localize with the dark holes in the FITC-gelatin (black arrows). Scale bars: $10 \mu \mathrm{m}$. 
A
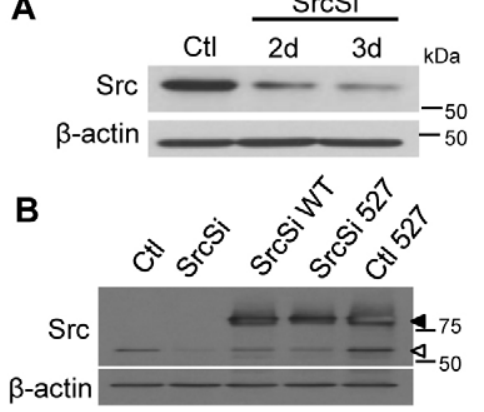

C

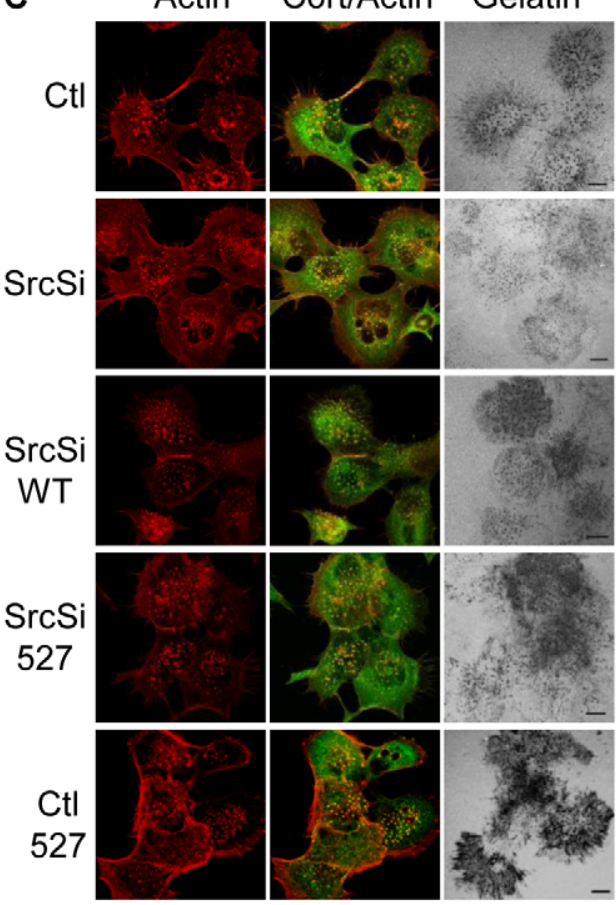

D
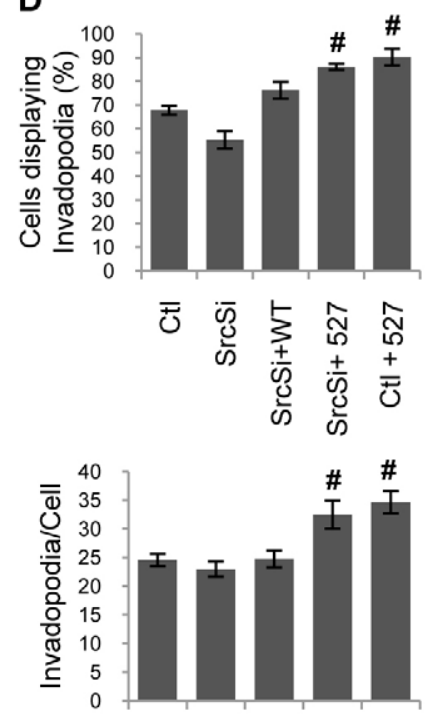

ฮ कु

ก

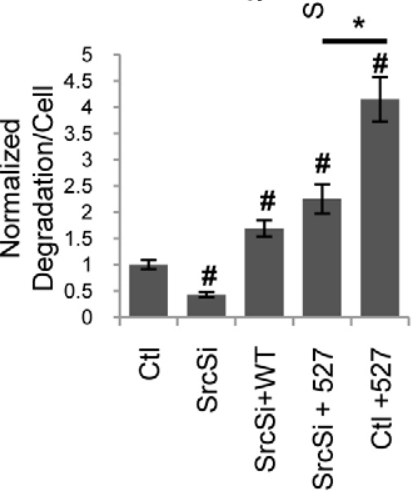

Fig. 2. Inhibition of endogenous Src expression decreases matrix degradation independently of changes in invadopodia number or increased Src activity. (A) UMSCC1 cells transfected with siRNA directed against $\mathrm{Src}(\mathrm{SrcSi})$ or a non-targeting siRNA $(\mathrm{Ctl})$ were evaluated for Src knockdown 2 and 3 days after transfection by anti-Src western blotting. $\beta$-actin blotting was used as a loading control. (B) Cell lysates from UMSCC1 cells transfected with non-targeting siRNA (Ctl) or SRC siRNA ( $\mathrm{SrcSi}$ ) alone, or in combination with cerulean-tagged SrcWT (WT) or Src-527F (527) were evaluated by immunoblotting. Lysates were probed with anti-Src and anti- $\beta$-actin antibodies. Filled arrowhead indicates exogenously expressed Src (WT or 527F), open arrowhead denotes endogenous Src. (C) Representative confocal images of UMSCC1 cells transfected with nontargeting siRNA $(\mathrm{Ctl})$ or $\mathrm{SrcSi}$ alone, or in combination with cerulean-tagged SrcWT (WT) or Src-527F (527). Cells were plated on FITC-gelatin-coated (pseudocolored white) coverslips for 10 hours and immunolabeled with TRITC-phalloidin (red) and cortactin (green). Scale bars: $10 \mu \mathrm{m}$. (D) Percentage of cells displaying invadopodia (top), the number of invadopodia per cell (middle) and the amount of matrix degradation per cell (bottom) were examined for each line evaluated in B. Data are presented as mean \pm s.e.m.; $\#$, statistically different from control $(P \leq 0.01)$; groups under the bar are statistically different $(P<0.01)$ in gelatin degradation compared with control cells (Ctl) (Fig. 2C, bottom panel). Rescue of WT Src expression in SrcSi cells ( $\mathrm{SrcSi}+\mathrm{WT}$ ) restored matrix degradation to levels above those of the control cells (1.7-fold) (Fig. 2C). Increases above control levels are presumably due to the additive effect of remaining endogenous Src from incomplete knockdown coupled with the modest overexpression of the WT Src construct (Fig. 2B).

We also examined the effect of silencing endogenous c-Src in the presence of constitutively active Src (Fig. 2B,C). Consistently with previous reports (Artym et al., 2006; Oser et al., 2009), expression of Src527F in control cells $(\mathrm{Ctl}+527)$ or $\mathrm{SrcSi}$ cells $(\mathrm{SrcSi}+527 \mathrm{~F})$ resulted in increases in the number of cells with invadopodia $(\sim 30 \%)$ and the number invadopodia per cell $(\sim 36 \%)$. Surprisingly, c-Src knockdown markedly blunted the increased degradation due to Src527F expression, a 2.5-fold ( $\mathrm{SrcSi}+\mathrm{Src527F}$ cells) increase compared with a 4.8 -fold $(\mathrm{Ctl}+527 \mathrm{~F})$ increase over control cells (Fig. 2C). These results demonstrate that constitutively active Src cannot completely rescue the role of endogenous c-Src in regulation of matrix degradation. Collectively, these data indicate that although increased Src activity enhances invadopodia formation, the presence of endogenous c-Src is required for optimal matrix degradation. Similar results were found in OSC19 cells (supplementary material Fig. S2).

\section{Constitutively active Src is sufficient to promote invadopodia formation but not ECM degradation in Src- null cells}

Complete c-Src knockdown in our HNSCC lines is technically problematic because additional Src family kinases (Yes and Fyn) with unknown functions in invadopodia biology are present and maintained in OSC19 and UMSCC1 cell lines treated with SrcSi (Fig. 3A). We therefore used Src-, Yes- and Fyn-deficient (SYF) fibroblasts to further evaluate the role of WT Src in invadopodia function. A GFP-tagged temperature-sensitive mutant of $\mathrm{v}-\mathrm{Src}$ (tsLa29-GFP) was generated and expressed in SYF cells to dynamically regulate Src activity and invadopodia formation. Src kinase activation occurs within 15 minutes when cells are switched from the non-permissive temperature $\left(41^{\circ} \mathrm{C}\right)$ to the permissive temperature $\left(35^{\circ} \mathrm{C}\right)$ (Fig. $\left.3 \mathrm{~B}\right)$. $\mathrm{v}$-Src inactivation occurs within 30 minutes when cells are shifted back to $41^{\circ} \mathrm{C}$. Activation of $\mathrm{v}-\mathrm{Src}$ leads to the phosphorylation of cortactin on Tyr421, indicating that tsLa29-GFP regulates phosphorylation of a known downstream Src target that is crucial for invadopodia assembly. In agreement with previous reports (Walker et al., 2007), invadopodia formation is induced when cells expressing tsLa29-GFP are switched to the permissive temperature (Fig. 3C). These invadopodia are enriched with active $\mathrm{v}-\mathrm{Src}$ and phosphorylated cortactin (Fig. 3C; 


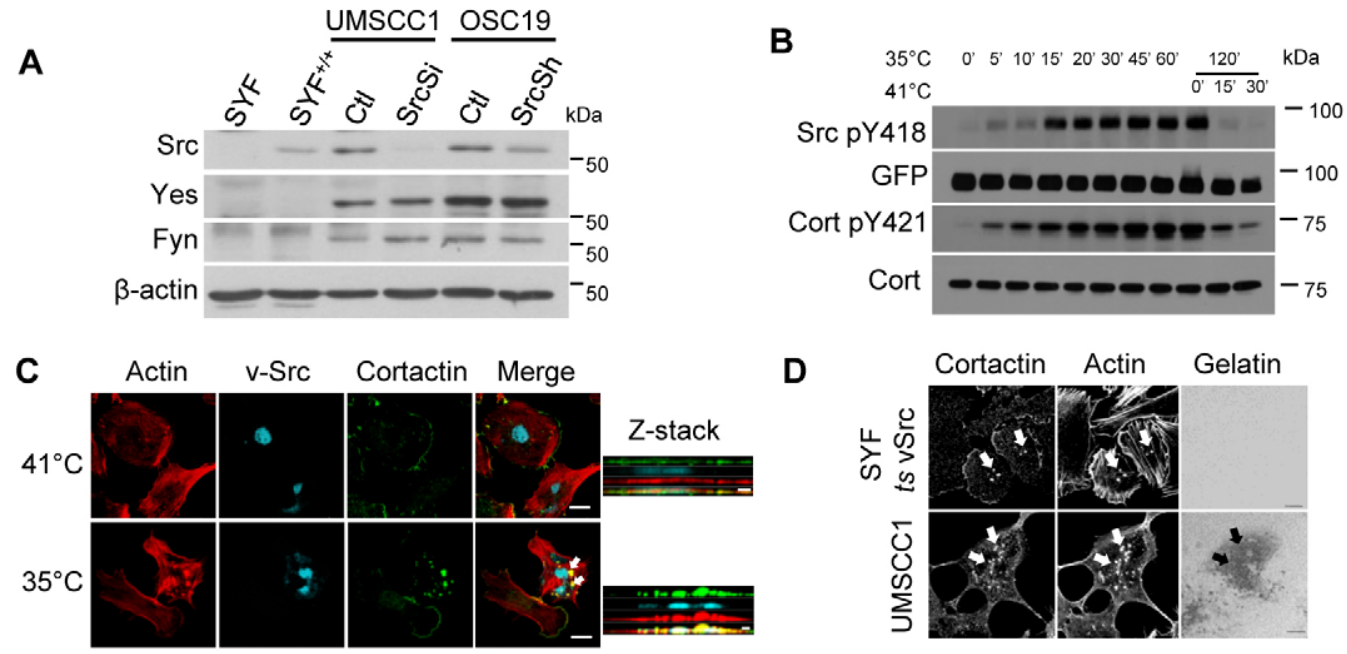

Fig. 3. Invadopodia produced by constitutively active Src in SYF fibroblasts fail to degrade ECM. (A) Src family kinase expression in HNSCC and fibroblast cell lines. Clarified cell lysates from SYF, SYF ${ }^{+/}$, UMSCC1 (treated with control and SrcSi), and OSC19 (expressing control vector and SRC shRNA) cells were resolved by SDS-PAGE and immunoblotted with anti-Src, anti-Yes, anti-Fyn and anti- $\beta$-actin antibodies. (B) Time course of tsLa29 v-Src activation and resulting cortactin phosphorylation. Cells transfected with tsLa29 were incubated at $35^{\circ} \mathrm{C}$ (permissive temperature) for the indicated times and were lysed or returned to $41^{\circ} \mathrm{C}$ (non-permissive temperature) for 15 or 30 minutes and analyzed for Src-pY ${ }^{418}$, GFP, cortactin, and cortactin-p ${ }^{421}$. (C) Invadopodia formation in cells expressing v-Src. SYF cells were transfected with tsLa29-GFP (pseudocolored light blue) and incubated at $41{ }^{\circ} \mathrm{C}$ or $35^{\circ} \mathrm{C}$ and labeled with TRITC-phalloidin (red) and cortactin (green). Cells were visualized by confocal microscopy and $z$-stack sectioning. Invadopodia are visible in $z$-stack images as actin- and cortactin-rich puncta that are several micrometers in length (white arrows). Scale bars: $10 \mu \mathrm{m}, 5 \mu \mathrm{m}$ (z-stacks). (D) Defective ECM degradation in SYF cells expressing v-Src. SYF cells transfected with tsLa29-v-Src and non-transfected UMSCC1 cells (positive control) were plated directly onto FITC-gelatin-coated coverslips. After 24 hours, cells were labeled with TRITC-phalloidin and anti-cortactin antibodies. White arrows indicate invadopodia and black arrows, areas of degraded matrix in C and D. Scale bars: $10 \mu \mathrm{m}$.

supplementary material Fig. S3A). As reported in other invadopodia-forming cell systems (Artym et al., 2006; Ayala et al., 2008; Clark et al., 2007; Webb et al., 2006), inhibition of cortactin expression using siRNA diminishes the ability of tsLa29-GFP to induce invadopodia formation at the permissive temperature (supplementary material Fig. S3B,C).

To evaluate the functionality of invadopodia in this system, we plated SYF cells on FITC-gelatin-coated coverslips to assay ECM degradation. Invadopodia induced by tsLa29-GFP in the Src-null fibroblasts fail to degrade the ECM at periods up to and beyond 48 hours, indicating they remain in a pre-invadopodia state (Fig. 3D). Experiments with an untagged $\mathrm{v}$-Src produced a similar result, ruling out improper activation or localization related to the addition of GFP. We also attempted to rescue invadopodia maturation in the SYF cells with tsLa29 v-Src. Manipulation of tsLa29 v-Src activity over the 24 hour incubation period by switching cells from permissive to the non-permissive temperatures did not result in invadopodia maturation (data not shown). These results demonstrate that constitutively active $\mathrm{v}$-Src activity is responsible for the induction of the initial phosphorylation cascade that drives recruitment of invadopodia components to form pre-invadopodia complexes, but these v-Src-induced complexes are insufficient to direct matrix degradation in SYF cells.

\section{Wild-type Src kinase is necessary for invadopodia maturation}

Based on our findings we hypothesized that either: (1) another ubiquitously expressed Src family kinase (Yes and/or Fyn) absent from SYF cells is required for invadopodia maturation in addition to active Src, or (2) WT Src or 'regulated' Src must also be present with active Src for degradation of ECM to occur. To test these hypotheses, we used a SYF cell line with two copies of WT Src genetically reintroduced to restore normal WT Src expression $\left(\mathrm{SYF}^{+/+}\right.$; Fig. 3A, Fig. 4A). The percentage of cells forming invadopodia and the percentage of invadopodia-forming cells degrading matrix were assessed following introduction of v-Src or Src527F. Transfection with activated Src constructs in the form of tsLa29 or Src527F induces mature matrix-degrading invadopodia in $\mathrm{SYF}^{+/+}$cells, in contrast to pre-invadopodia formation in SYF cells (Fig. 4B). Approximately 55\% of $\mathrm{SYF}^{+/+}$cells forming invadopodia contained invadopodia that actively degraded matrix, compared with $2 \%$ of SYF cells (Fig. 4C). There was no difference in the percentage of cells forming invadopodia (pre and mature) in SYF or SYF ${ }^{+/+}$cells expressing Src527F (Fig. 4C), which is similar to results in UMSCC1 cells (Fig. 2C). Also, the level of general phosphotyrosine-containing proteins localized to invadopodia was unchanged in SYF527F and SYF527F ${ }^{+/+}$cells (Fig. 4B). Taken together, these results suggest that catalytically active Src alone promotes the assembly of pre-invadopodia complexes and targets tyrosine phosphorylation of proteins within these structures, but SrcWT is necessary for pre-invadopodia maturation required to induce ECM degradation.

To confirm these findings, WT Src expression was transiently restored in SYF cells and assayed for ECM degradation. Monitoring of the coexpression of Src527F and SrcWT was achieved by creating C-terminal linker fusions with mCherry (Src527FmCherry) and cerulean (Src-Cer) fluorescent proteins (supplementary material Fig. S4). Transfection efficiency of the co-transfected Src constructs was consistently greater than $90 \%$ and imaging revealed that nearly all SYF cells expressed both Src alleles (supplementary material Fig. S5A). In SYF cells expressing SrcWT alone, Src had a perinuclear localization that was consistent with previous reports (Sandilands et al., 2004) (supplementary material Fig. S4B,C). Coexpression of Src527F with WT Src 
A

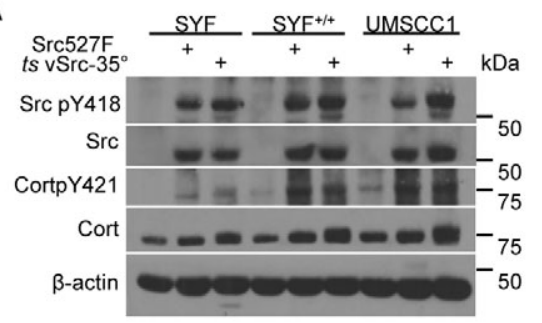

C

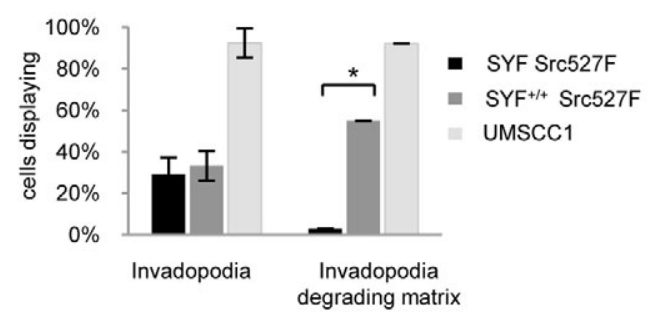

B

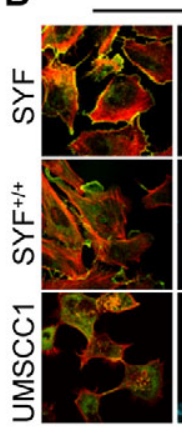

Actin/
NT

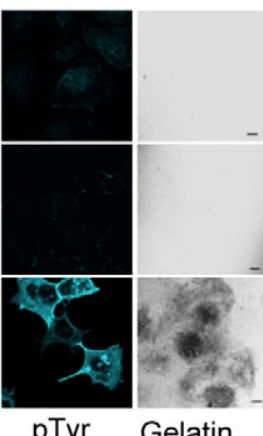

pTyr Gelatin
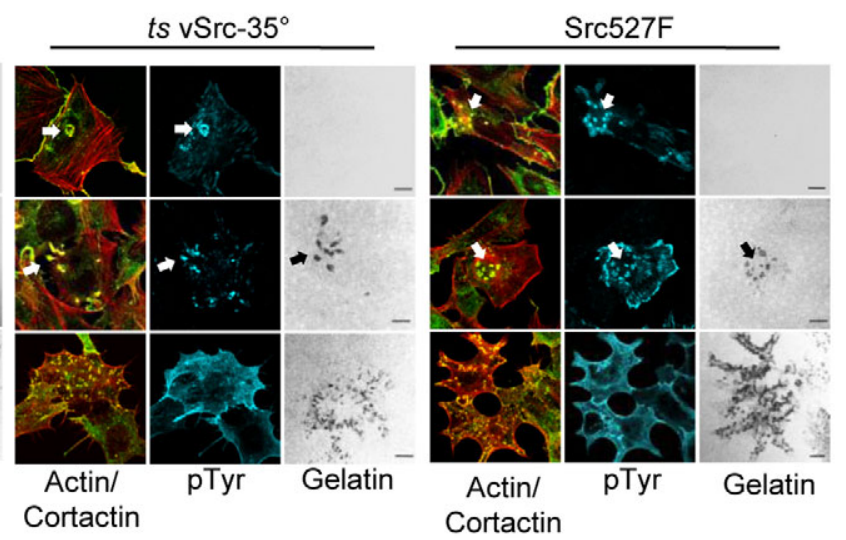

Fig. 4. WT Src rescues invadopodia maturation in cells expressing constitutively active Src. (A) SYF, SYF ${ }^{+/+}$and UMSCC1 cells that were non-transfected (NT), transfected with Src-527F or ts-v-Src, were lysed, resolved by SDS-PAGE and immunoblotted with anti-Src-pY418, anti-Src clone EC10 (only recognizes avian Src), anti-cortactin-pY421, anti-cortactin (4F11), and anti- $\beta$-actin antibodies. (B) SYF, SYF ${ }^{+/}$and UMSCC1 cells that were non-transfected (NT), transfected with SRC-527F, or tsLa29-v-Src were plated onto FITC-conjugated gelatin coverslips and evaluated by confocal microscopy. Cells were labeled with TRITCphalloidin (red), cortactin (green), and anti-phosphotyrosine (light blue) antibodies. Invadopodia are identified by the yellow aggregates in the merged image of actin and cortactin in cells containing phosphotyrosine. In addition, degrading (mature) invadopodia localize with the dark holes (arrows) in the FITC-gelatin (white). (C) Quantification of the percentage of cells forming invadopodia structures (actin and cortactin aggregates, left), and the percentage of invadopodiaforming cells that contain matrix degradation (mature invadopodia, right). Data are represented as mean \pm s.d.; $* P \leq 0.05$.

results in recruitment of WT Src to invadopodia where it colocalizes with Src527F (Fig. 5A). In addition, cells coexpressing these constructs regain the ability to degrade ECM (Fig. 5B,C). To further verify the functional requirement for WT Src in invadopodia maturation, we conducted WT-Src-specific staining of SYF cells containing Src527F. WT Src colocalizes with cortactin to areas of ECM degradation, further demonstrating that Src localizes to mature invadopodia (Fig. 5D, top panels). In addition, direct visualization of WT Src-Cer and Src527F-mCherry in SYF cells demonstrates a concentration of $\mathrm{Src}-\mathrm{Cer}$ at areas of ECM degradation, with $\mathrm{Src} 527 \mathrm{~F}-\mathrm{mCh}$ localized to the same vicinity (Fig. 5D, bottom panels).

Since the localization of endogenous Src to invadopodia is required for invadopodia maturation, we determined whether catalytically inactive Src could substitute for WT Src and rescue ECM degradation in SYF cells. This result would suggest that two separate and distinct pools of Src (constitutively active and kinaseinactive) are necessary and sufficient for maturation. To test this hypothesis, constitutively active Src527F-mCherry was coexpressed with a cerulean-tagged kinase-inactive Src (Src295MCer) in SYF cells. Similarly to WT Src, Src295M was largely perinuclear when expressed alone in SYF cells (supplementary material Fig. S4B,C), but was recruited to invadopodia when coexpressed with Src527F (Fig. 5A). However, Src295M failed to rescue invadopodia maturation (Fig. 5B,C), indicating that catalytically inactive Src does not substitute for WT Src function. Since constitutively active and kinase-dead Src cannot support invadopodia maturation, this suggests that WT Src kinase activity is dynamically regulated to promote invadopodia maturation. Similarly, cell staining for active Src (pY418) localized to preinvadopodia in SYF cells and mature invadopodia in $\mathrm{SYF}^{+/+}$cells (Fig. 5E), ruling out the possibility that catalytically active Src initially drives invadopodia assembly, then is inactivated and remains inactive during maturation. Taken together, these results demonstrate that regulated WT Src kinase activation and inactivation within pre-invadopodia complexes is necessary to govern the downstream signaling events required for invadopodia maturation and ECM degradation.

\section{Regulated Src activity directs cortactin phosphorylation dynamics to control invadopodia maturation}

In proposed models of invadopodia maturation, dynamic cortactin tyrosine phosphorylation is required for pre-invadopodia maturation (Oser et al., 2009). To identify a potential mechanism of invadopodia maturation affected by regulated $\mathrm{Src}$ activation and inactivation, we assessed the role of Src kinase activity on cortactin phosphorylation. In the SYF/SYF ${ }^{+/+}$system, cortactin phosphorylated on Tyr421 localized to pre-invadopodia and mature invadopodia (Fig. 6A), suggesting that cortactin phosphorylation is important in pre-invadopodia assembly and invadopodia maturation. This is consistent with a well-described role for cortactin in invadopodia maturation in other systems (Artym et al., 2006; Clark et al., 2007; Oser et al., 2009). Stable cell lines were generated expressing endogenous levels of WT cortactin (control) 

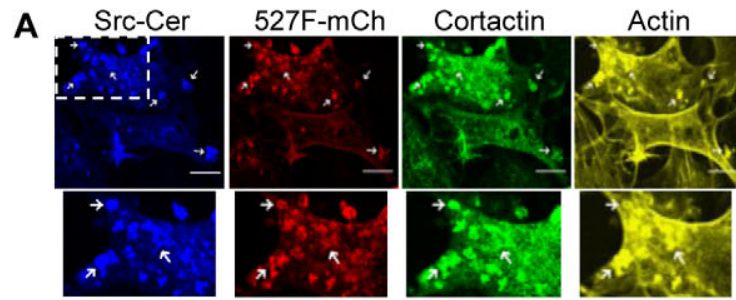

Src295M-Cer
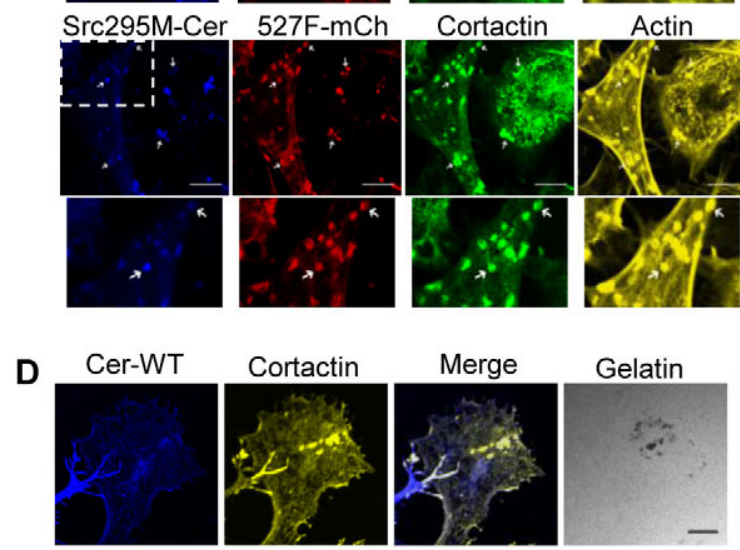

Gelatin
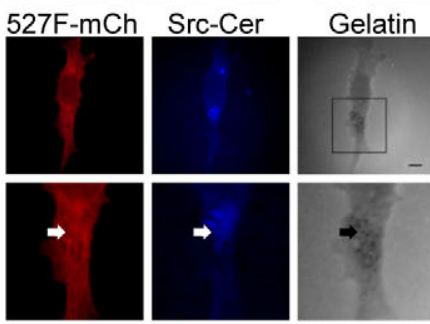

B

$\mathrm{SYF}^{+1+}$

527F-mCh
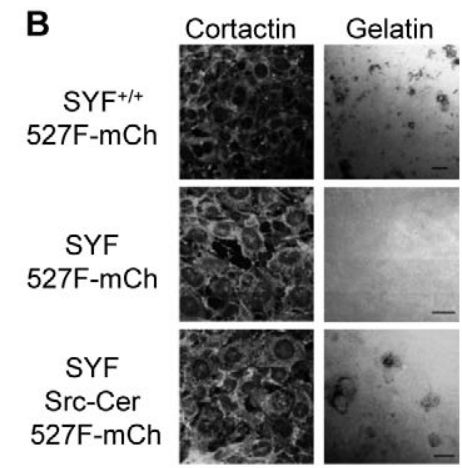

SYF

Src295M-Cer 527F-mCh

E

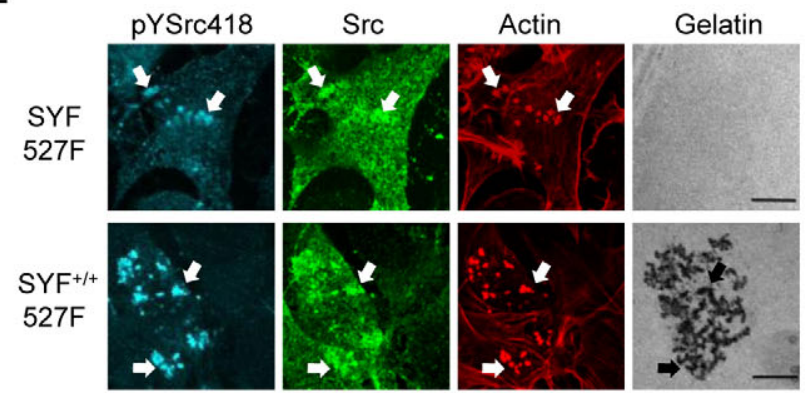

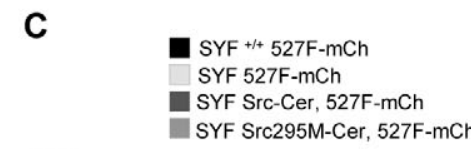

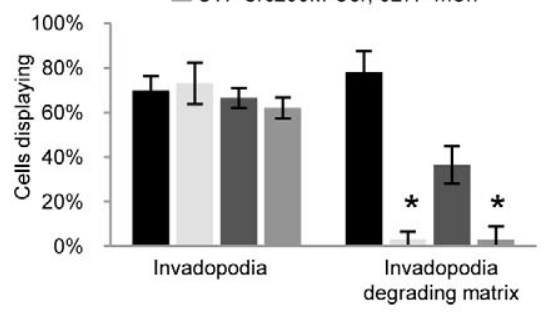

Fig. 5. Expression of regulated Src is necessary for ECM degradation at invadopodia. (A) Confocal imaging of SYF cells coexpressing Src-Cerulean and Src527F-mCherry, or Src-295M-Cerulean and Src527F-mCherry. Cells were immunolabeled with anti-cortactin and Alexa-Flour-647-labeled phalloidin. Dashed boxes represent enlarged image regions shown below. Arrows denote invadopodia. (B) Representative fields of SYF and SYF ${ }^{+/+}$cells expressing Src527FmCherry, SYF cells coexpressing Src527F-mCherry and Src-Cerulean, or SYF cells coexpressing Src527F-mCherry and Src295M-Cerulean. Cells were plated on FITC-gelatin-coated coverslips for 24 hours and immunolabeled with anti-cortactin. Areas of gelatin degradation and clearing appear black against the pseudocolored white background. (C) Percentage of cells with forming invadopodia (actin and cortactin aggregates, left), and percentage of invadopodia-forming cells with degraded matrix (mature invadopodia, right) from the experimental conditions shown in B. Data are represented as mean $\pm \mathrm{s} . \mathrm{d}$.; $* P \leq 0.05$. (D) Confocal imaging (top) or swept-field imaging (bottom) of SYF cells coexpressing c-Src527F and Src-Cerulean. Cells were plated on FITC-gelatin-coated coverslips and immunolabeled with anti-cerulean (dark blue) and anti-cortactin (yellow) (colocalization appears white in merged image; top panel) or directly imaged for Src527F-mCherry (red) and Src-Cerulean (dark blue) (bottom panels). Arrow indicates colocalization of Src 527F-mCherry and WT Src-Cerulean at sites of gelatin degradation. (E) Confocal imaging of Src activity in SYF and SYF ${ }^{+/+}$cells expressing Src527F. Cells were incubated on FITC-gelatin-coated coverslips for 24 hours, fixed and immunolabeled with TRITC-phalloidin (red), anti-pY418 Src (light blue) and anti-Src (green) antibodies. White arrows denote invadopodia; black arrows, matrix degradation. Scale bars: $20 \mu \mathrm{m}$ (B), $10 \mu \mathrm{m}$ (A,D,E).

or a cortactin mutant in which the three Src-targeted tyrosine residues $(421,470,486)$ are mutated to phenylalanine $\left(\mathrm{SYF}^{+/+}\right.$ CortTYM) (Fig. 6B). Endogenous murine cortactin was silenced with siRNA (CortSi, knockdown $>90 \%$ ), resulting in the exclusive expression of WT or mutant human cortactin (Fig. 6C). Wild-type control and CortTYM SYF ${ }^{+/+}$cell lines treated with siRNA to knock down cortactin were transfected with Src527F to promote invadopodia formation. Expression of WT human cortactin rescued the inhibitory effects of cortactin knockdown on invadopodia formation, resulting in the formation of mature invadopodia $(\sim 60 \%$ of total cells with invadopodia, Fig. $6 \mathrm{D}, \mathrm{E})$. However, only $\sim 10 \%$ of $\mathrm{SYF}^{+/+}$CortTYM cells produced degrading invadopodia. Consistent with previous reports (Oser et al., 2009), there were no differences observed in the percentage of cells forming actin or cortactin aggregates in control and CortTYM SYF SFlls, $^{+/+}$cell demonstrating that cortactin is targeted to pre-invadopodia independently of tyrosine phosphorylation. However, expression of mutant cortactin in $\mathrm{SYF}^{+/+}$cells completely blocked the upstream function of WT Src, rendering the $\mathrm{SYF}^{+/+}$Src527F cells with a degradation profile that was similar to that seen in cells lacking WT Src (SYF Src527F, Fig. 4B,C). These results indicate that there is differential phosphotyrosine signaling in SYF cells with WT Src that supports invadopodia maturation downstream of constitutively active Src.

\section{Cells forming pre- and mature invadopodia have distinct phosphotyrosine signatures}

To examine whether distinct tyrosine phosphorylation signatures occur in cells that form pre-invadopodia and mature invadopodia, we conducted a non-biased, comprehensive and quantitative SH2domain screen (Machida et al., 2007) to identify differences in potential phosphotyrosine-binding proteins under conditions of 
A
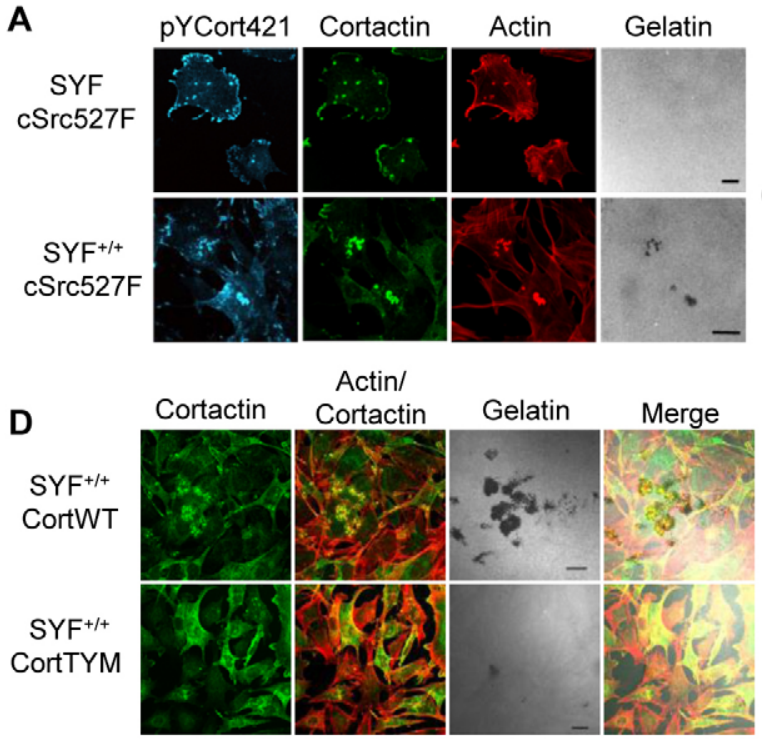

B
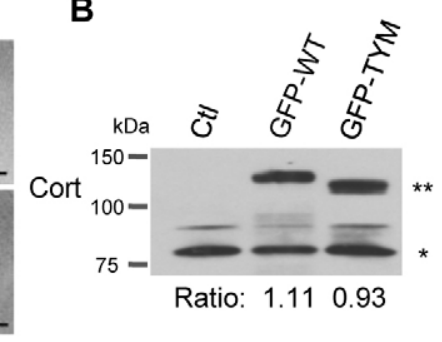

C

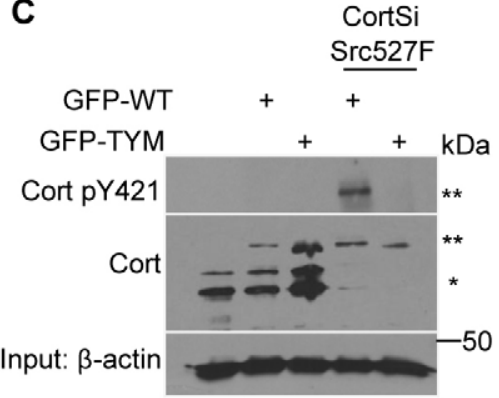

E

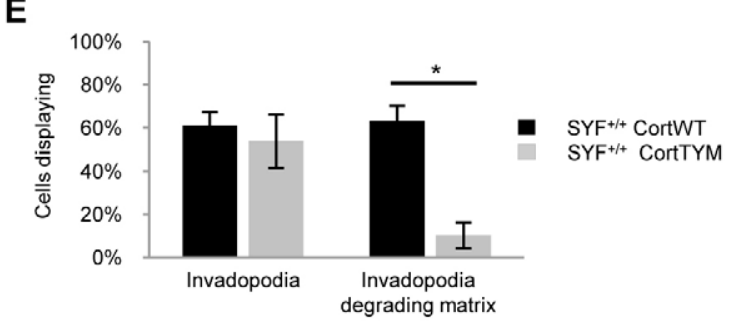

Fig. 6. WT Src regulates cortactin phosphorylation during invadopodia maturation. (A) SYF and $\mathrm{SYF}^{+/+}$cells expressing Src527F were incubated on FITCgelatin-coated coverslips for 24 hours, fixed and immunolabeled with TRITC-phalloidin (red), human anti-pY421 cortactin (light blue) and anti-cortactin (green) antibodies. Note that cortactin is phosphorylated in pre-invadopodia and in mature invadopodia, as defined by the absence or presence of matrix degradation. (B) Validation of GFP-tagged cortactin expression in stable cell lines. Cellular extracts from SYF or SYF ${ }^{+/+}$cells stably expressing GFP-CortWT or GFPCortTYM were resolved by SDS-PAGE and immunoblotted with an anti-cortactin (4F11) antibody. *, endogenous cortactin; **, GFP-tagged cortactin (the slight mobility disparity is due to the size difference in AcGFP and EGFP tags); ratios of exogenous (WT and TYM) to endogenous cortactin is depicted under the blot. (C) Clarified lysates from SYF ${ }^{+/+}$GFP-CortWT and SYF ${ }^{+/+}$GFP-CortTYM cells were transfected with Cttn siRNA alone or in combination with Src527F. Cortactin was immunoprecipitated with the anti-cortactin (4F11) antibody, immunocomplexes resolved by SDS-PAGE and immunoblotted with human anti-pY421 cortactin and anti-cortactin (4F11) antibodies. Total cell lysates were immunoblotted with anti- $\beta$-actin as a loading control. (D) SYF ${ }^{+/+}$cells stably expressing human GFP-CortWT or GFP-Cort TYM were transfected with murine cortactin-targeted siRNA to eliminate endogenous cortactin expression. Two days later cells were transfected with Src527F and plated on FITC-gelatin-coated coverslips for 24 hours to promote gelatin degradation. (E) Cells were assessed for the percentage of cells forming invadopodia (actin and cortactin aggregates) and the percentage of invadopodia-forming cells with matrix degradation. Data are represented as mean \pm s.d., ${ }^{*} P<0.05$. Scale bars: $10 \mu \mathrm{m}(\mathrm{A}), 20 \mu \mathrm{m}(\mathrm{B})$.

pre-invadopodia and mature invadopodia formation (Fig. 7; supplementary material Fig. S6). Non-transfected SYF and SYF ${ }^{+/+}$ cells had minimal differences in SH2-domain binding profiles. Expression of Src527F in either cell type enhanced overall SH2 domain signal-binding intensity, indicating a broad increase in phosphotyrosine signaling and creation of new SH2-domain docking sites. This result was anticipated with expression of constitutively active Src. However, cells that form pre-invadopodia (SYF Src527F) had distinct differences in their SH2 binding intensity from cells that form functional mature invadopodia $\left(\mathrm{SYF}^{+/+} \mathrm{Src527 \textrm {F } )}\right.$ (bottom row, difference). The variations in binding intensity that arise in SYF and $\mathrm{SYF}^{+/+}$cells expressing Src527F indicate a fundamental mechanistic difference in the concentration of phosphotyrosine binding sites for several SH2domain-containing proteins that are likely to have key roles in invadopodia maturation. Interestingly, tyrosine-phosphorylated cortactin is known to interact with several high-intensity 'hits', including Arg, Abl (Boyle et al., 2007), Fer (El Sayegh et al., 2005), Crk (Bougneres et al., 2004) and Nck (Tehrani et al., 2007), consistent with its role in invadopodia maturation. Experiments to elucidate additional proteins involved in these signaling complexes are currently underway.

\section{Discussion}

In this study, we investigated the role of endogenous or WT c-Src, and the interplay between constitutively active Src and cellular Src in invadopodia formation. Previous studies on Sre in invadopodia formation have exclusively manipulated Src activity in cells containing WT c-Src. Tumor cells that form spontaneous invadopodia presumably have upstream oncogenic signals such as overactivation or overexpression of epidermal growth factor receptor (EGFR), which drives c-Src activation (Xue et al., 2006). Accordingly, increased Src kinase activity (through overexpression of constitutively active Src, or overexpression of WT Src) in tumor cells that form spontaneous invadopodia is associated with increased invadopodia formation and matrix degradation (Artym et al., 2006; Oser et al., 2009). Unlike previous reports (Oser et al., 2009), we did not find that tumor cells overexpressing constitutively active Src had less degradation per invadopodia than spontaneous invadopodia formed in control cells (Fig. 2; supplementary material Fig. S2). However, these differences might be attributed to a much larger capacity for invadopodia formation in UMSCC1/OSC19 cells compared with MtLn3 cells ( $\sim 25$ vs $\sim$ two invadopodia formed in control cells, respectively). In HNSCC cells and Src-null fibroblasts, we propose that constitutively active Src acts as an oncogenic 'trigger' that promotes pre-invadopodia formation, whereas WT Src acts downstream to direct invadopodia stability and maturation. Although models of spontaneous invadopodia formation are invaluable to the field, our finding that Src cycling is essential for invadopodia maturation could only be completely uncovered through the use of the SYF/SYF ${ }^{+/}$system.

In this study, we show that WT Src is indispensable for invadopodia maturation driven by elevated Src activity. We hypothesize that distinct, temporally and spatially regulated Src 


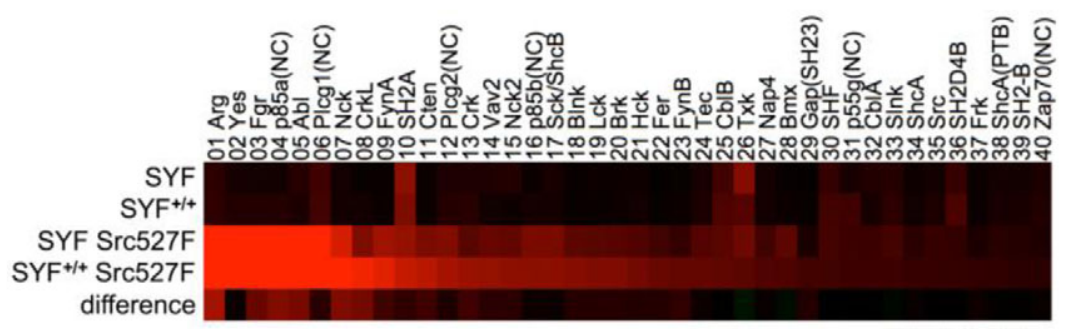

8888888
Fig. 7. Enhanced binding of $\mathrm{SH} 2$ domains binding in SYF $^{+/+}$cells expressing Src527F. In vitro SH2 and PTB domain-binding profiles for SYF, $\mathrm{SYF}^{+/+}$cells, and SYF and $\mathrm{SYF}^{+/+}$cells expressing Src527F. Binding of SH2 or PTB domains to cell lysates are shown as a heat map where intensity of red indicates strength of binding. Domain names are on the top row, with a rank order of binding to $\mathrm{SYF}^{+/+}$ Src $527 \mathrm{~F}$ to denote potential importance in invadopodia maturation. Sample labels are on the left side.

Difference=Signal[SYF ${ }^{+/+}$Src527F]-Signal[SYF Src527F]. function is necessary to regulate the phosphorylation of cortactin. It is likely that Src regulates other scaffolding proteins that are important in invadopodia stability and maturation, such as dynamin (Caldieri et al., 2008), Tks5 (Seals et al., 2005), IQGAP1 (SakuraiYageta et al., 2008) and paxillin (Bowden et al., 1999). It is possible that Src is activated before its localization with downstream actin-associated substrates in pre-invadopodia. Once preinvadopodia assembly is complete, Src is inactivated [presumably by C-terminal Src kinase (CSK) acting on pY527 (Okada et al., 1991)] and released to regulate additional proteins involved in actin dynamics and MMP delivery to invadopodia. In this way, Src might act as a regulator of several sequentially coordinated proteininteraction events that direct the diverse array of cellular processes at invadopodia, including actin assembly, membrane trafficking and ECM degradation.

It is known that invadopodia produced by cancer cells and transformed fibroblasts use similar signaling pathways and contain many of the same proteins as podosomes produced by osteoclasts, dendritic cells and macrophages (Linder, 2009). SRC-null osteoclasts have been used to study the role of Src in podosome assembly and dynamics (Destaing et al., 2008). WT Src and Src527F were both able to restore normal podosome organization, whereas Src295M did not. These data in podosomes support our findings in invadopodia, but since this report did not address the functionality of these cells to degrade ECM, it is not known whether WT Src is needed for maturation in this system. These results are particularly interesting because Src has been suggested to act as a 'molecular switch' to regulate dynamin-Cbl signaling complexes (Bruzzaniti et al., 2005) and as a protein with an important (kinase independent) adaptor function (Bruzzaniti et al., 2009) in osteoclast podosomes. Manipulation of Src activity in WT osteoclasts also suggest a dual function for Src in the regulation of actin dynamics through cortactin phosphorylation in podosome assembly and subsequent maturation into more highly organized structures known as sealing zones (Luxenburg et al., 2006).

We identify cortactin as a key regulator of invadopodia maturation downstream of WT Src activity. We show that constituitively active Src is sufficient to target cortactin to preinvadopodia (Fig. 3C, Fig. 4B), but cortactin is targeted independent of its tyrosine phosphorylation (Fig. 6D,E). This is in agreement with previous reports demonstrating that dephosphorylated cortactin acts as a scaffold to recruit Arp2/3, N-WASp and cofilin (Oser et al., 2009). Interestingly, cortactin phosphorylated at Tyr421 is enriched in pre-invadopodia (SYF Src527F) and in matrixdegrading mature invadopodia $\left(\mathrm{SYF}^{+/+} \mathrm{Src527F}\right)$ (Fig. 6A; supplementary material Fig. S3A). In addition, tyrosine phosphorylation of cortactin is required for invadopodia maturation and ECM degradation (Fig. 6D,E) (Ayala et al., 2008; Desmarais et al., 2009; Webb et al., 2007). These data suggest that phosphorylation of cortactin occurs before ECM degradation. However, in our SYF/SYF ${ }^{+/+}$model this initial cortactin phosphorylation is not sufficient to drive maturation in the absence of WT Src, suggesting that cortactin must be dephosphorylated before maturation can occur. Our proposed mechanism of dynamic Src activity on cortactin phosphorylation in invadopodia maturation is consistent with the model recently proposed by the Condeelis laboratory (Oser et al., 2009), whereby cyclical cortactin phosphorylation regulates actin polymerization and invadopodia stabilization.

Here, we show that Src activation and inactivation regulates cortactin phosphorylation during invadopodia maturation. Future studies will be required to determine whether Src kinase is directly responsible for the initial phosphorylation of cortactin to promote invadopodia assembly or in subsequent $\operatorname{step}(\mathrm{s})$ to regulate maturation (Huang et al., 1998). Fer (El Sayegh et al., 2005) and Abl family kinases (Boyle et al., 2007) are also present in SYF cells and might act downstream of Src to regulate one or more of these steps. It also follows that tyrosine phosphatases are critically important in the regulation of invadopodia maturation, because cortactin and other possible targets downstream of Src kinase activity in our system require dynamic cycles of phosphorylation and dephosphorylation to drive ECM degradation. Protein tyrosine phosphatase $1 \mathrm{~B}$ is a good candidate for this process because it is known to regulate Src in invadopodia dynamics (Cortesio et al., 2008) and has subsequently been shown to regulate cortactin dephosphorylation (Stuible et al., 2008). Nonetheless, we show that WT Src kinase is the critical upstream regulator of other downstream kinases and phosphatases important to these processes.

Invadopodia maturation is associated with the delivery of MMPs to invadopodia sites (Artym et al., 2006; Clark et al., 2007). MT1MMP is cited as the key MMP that regulates ECM degradation at invadopodia (Poincloux et al., 2009) and Src kinase activity is known to regulate phosphorylation of MT1-MMP and proteins associated with its trafficking to the cell membrane (Nyalendo et al., 2008; Nyalendo et al., 2007). Future studies should address whether WT Src is necessary to control trafficking to, or activation of MT1-MMP at invadopodia. These studies are further warranted because it has been proposed that a major role of cortactin in invadopodia function involves the targeting and delivery of MMPs to invadopodia to enhance ECM degradation (Clark and Weaver, 2008; Clark et al., 2007).

This report identifies a dominant oncogene that requires the proto-oncogenic complement to reach its full spectrum of transforming functionality. Unlike tumor suppressor proteins, which often require loss of non-mutated alleles, little is known about the potential effect of the presence or absence of proto-oncogenes on their oncogenic counterparts during tumorigenesis. A function in Ras-induced tumorigenesis has been described for Ras proto- 
oncogenes (Singh et al., 2005), where WT Ras has been reported to regulate the functioning of oncogenic Ras with regards to cell proliferation. Our results are consistent with those from the majority of solid human tumors that contain elevated c-Src expression or specific activity due to hyperactivation of upstream regulatory pathways, rather than activating point mutations (Yeatman, 2004). To our knowledge, this is the first description of such a finding. Similar mechanisms might be required for other transforming kinases involved in driving tumor progression.

\section{Materials and Methods}

\section{Cell culture}

HNSCC cell lines UMSCC1, FADU and OSC19 were obtained from Jeffery Myers (M. D. Anderson Cancer Center, Houston, TX). These lines along with UMSCC2 (Thomas Carey, University of Michigan, Ann Arbor, MI), 1483 (David Raben, University of Colorado Denver, Aurora, CO) and MSK921 (Peter Sacks, New York University, New York, NY) were cultured as previously described (Rothschild et al., 2006). SYF $\left(\mathrm{Src}^{-/} \mathrm{Yes}^{-/-} \mathrm{Fyn}^{-/-}\right)$and $\mathrm{SYF}^{+/+}\left(\mathrm{Src}^{+/+} \mathrm{Yes}^{-/-} \mathrm{Fyn}^{-/-}\right)$cells were obtained from the American Type Culture Collection. Control non-silencing and human SRCtargeted siRNA (5'-AAACTCCCCTTGCTCATGTACTT-3') were from Dharmacon. OSC19 cells stably expressing vector control or $S R C$-targeted shRNA were created by infection with control or human Src specific lentivirus (Santa Cruz) and cultured according to the manufacturer's instructions. SYF or $\mathrm{SYF}^{+/+}$cells stably expressing GFP-tagged human cortactin \{WT or Y421F, Y466F, Y482F [triple point mutant (TPM)] $\}$ were created using the Flp- $\mathrm{In}^{\mathrm{TM}}$ system (Invitrogen). These lines were transfected with siRNA targeting murine cortactin (Cttn) (5'-GCTTCGAGAGAATGTCTTC-3') (siCTTN, Dharmacon). For transient transfections, $3 \times 10^{6}$ cells were incubated with $2 \mu \mathrm{g}$ plasmid construct or siRNA. Fibroblast lines were transfected with the Nucleofector I device (Amaxa Biosystems) and HNSCC lines were transfected with TurboFect ${ }^{\mathrm{TM}}$ transfection reagent (Fermentas).

\section{Immunofluorescence labeling and confocal microscopy}

Cells were plated on FITC-gelatin (Sigma)-coated coverslips as described (Artym et al., 2006) for 12-24 hours. Cells were fixed with fresh $4 \%$ formaldehyde and permeabilized with $0.4 \%$ Triton X-100 in PBS. Primary antibodies were diluted with $5 \%$ BSA in PBS. Antibodies used were: cortactin 4F11, cortactin EP1922Y (Novus Biologicals), human cort-pY421 (Ammer et al., 2009), Src-pY418 (Biosource), Src GD11 (Upstate), GFP/Cerulean 3E6 (Invitrogen), pTyr-100 (BD Biosciences), Alexa Fluor 405 goat anti-rabbit and Alexa Fluor 647 goat anti-mouse (Molecular Probes). F-actin was labeled with Rhodamine-conjugated phalloidin (Molecular Probes). Cells were mounted in Fluoromount-G (Southern Biotech) and imaged with a Zeiss LSM510 confocal microscope using AIM software (Carl Zeiss MicroImaging). A Nikon Swept-Field using Nikon Elements software (Nikon Instruments) was used for imaging of Src-Cer.

\section{Invadopodia and matrix-degradation assays}

Cells with invadopodia were identified by the presence of at least one actin and cortactin aggregate within the cell $(n \geq 300)$. The number of invadopodia per cell was calculated by counting the number of actin and cortactin aggregates within invadopodia forming cells $(n \geq 150)$. Degradation per cell area was analyzed using ImageJ as described previously (Clark et al., 2007). Cells with invadopodia-degrading matrix were identified by at least one actin and cortactin aggregate colocalizing with a 'dark hole' that corresponded to degraded FITC-matrix $(n \geq 50)$. Data were pooled from several independent experiments; $n$ represents the number of cells analyzed within each experimental group.

\section{Antibodies and western blotting}

Western blotting of cell lysates was conducted as described (Rothschild et al., 2006). The following antibodies were used: 4F11, Src clone GD11 (Upstate); $\beta$-actin (Calbiochem); Living Colors GFP clone JL-8 (BD); Cort-pY421, Src-pY418 (Biosource); avian Src clone EC10 (Millipore) and Yes, Fyn (Cell Signaling).

\section{Plasmids}

The Src-GFP linker constructs (WT, 527F, and 295M) were a gift from Margaret Frame (The Beatson Institute for Cancer Research, Glasgow, UK). Substitution of green fluorescent protein (GFP) with cerulean or mCherry fluorescent protein was accomplished through digestion of Src-pEGFP-N1, pmCherry-C1, and mCerulean$\mathrm{C} 1$ fluorescent vectors with AgeI and BrsGI. The resulting mCherry and mCerulean fragments ( $\sim 700 \mathrm{bp})$ were ligated into the GFP-digested Src-containing pEGFP-N1 vector (BD). Temperature-sensitive v-Src (tsLA29) was subcloned from pCMVtsLA29 vector into EGFP-N1 using unique EcoRI and BamHI restriction sites.

\section{Human cortactin constructs.}

A single-stranded primed cDNA library (Invitrogen) was used for cloning human cortactin (CTTN) cDNA. The cDNA was PCR amplified to produce a 965 bp KpnIHincII fragment and a 688 bp HincII-EcoRI fragment. Fragments were ligated into
pcDNA3FLAG2AB that had been digested with $K p n \mathrm{I}$ and $E c o$ RI, to generate the 1653 bp full-length CTTN cDNA. The CTTN triple tyrosine mutant (TYM) was generated using the QuikChange kit with primers designed to alter codons 421, 470 and 486 from tyrosine to phenylalanine and confirmed by DNA sequencing. WT and TYM CTTN cDNAs were subsequently amplified as EcoRI-KpnI fragments and subcloned into pEGFP-N1 (WT) or pAcGFP-N1 (TYM). GFP-CTTN WT and TYM fragments were amplified by PCR and subcloned into pEF5/FRT/V5-D-TOPO (Invitrogen) and stable SYF and SYF ${ }^{+/+}$cell lines generated using the Flp-In system (Invitrogen) according to the manufacturer's instructions.

\section{Immunoprecipitation}

Cells were lysed in NP40 Buffer (20 mM HEPES-KOH, pH 7.8, $50 \mathrm{mM} \mathrm{KCl,} 1 \mathrm{mM}$ EDTA and $1 \% \mathrm{NP} 40)$. Anti-cortactin $(4 \mathrm{~F} 11,5 \mu \mathrm{g})$ was incubated with $0.5 \mathrm{mg}$ clarified lysates for 2 hours at $4^{\circ} \mathrm{C}$, then incubated with $40 \mu \mathrm{l}$ Protein $\mathrm{A} / \mathrm{G}$ Beads (Thermo Scientific) for 1 hour at $4^{\circ} \mathrm{C}$. Immune complexes were collected by centrifugation, washed twice with NP40 Buffer, separated by SDS-PAGE and western blotted with antibodies as described.

\section{SH2 and PTB binding assay}

SH2 and PTB domain binding assays were performed as described (Dierck et al., 2009; Machida et al., 2007). Briefly, SYF cell lysates were spotted in duplicate on a nitrocellulose membrane in register with the wells of a 96-well chamber plate. Each well was separately incubated with purified GST-SH2 or GST-PTB domains $(\sim 100 \mathrm{nM})$ for 2 hours. Probe binding was detected by enhanced chemiluminescence (ECL) (Perkin Elmer) and digitally captured (Kodak Image Station). Two independent experiments were performed in duplicate, providing four quantifiable data points for each probe. The array images were background-subtracted and the integrated density of each spot was measured using ImageJ (v1.40).

\section{Statistical analysis}

Differences in mean values between groups were evaluated using a Student's $t$-test (two groups) or a one-way ANOVA (multiple groups) followed by Scheffe post-hoc testing.

The contributions of the West Virginia University Microscope Imaging Facility, Mary Babb Randolph Cancer Center, are gratefully acknowledged. This work was supported by National Institute of Health grants P20 RR16440 and R01 DE014578 to S.A.W. Deposited in PMC for release after 12 months.

Supplementary material available online at http://jcs.biologists.org/cgi/content/full/123/22/3923/DC1

\section{References}

Ammer, A. G., Kelley, L. C., Hayes, K. E., Evans, J. V., Lopez-Skinner, L. A., Martin, K. H. and Weed, S. A. (2009). Saracatinib impairs head and neck squamous cell carcinoma invasion by disrupting invadopodia function. J. Cancer Sci. Ther. 1, 52-61. Artym, V. V., Zhang, Y., Seillier-Moiseiwitsch, F., Yamada, K. M. and Mueller, S. C. (2006). Dynamic interactions of cortactin and membrane type 1 matrix metalloproteinase at invadopodia: defining the stages of invadopodia formation and function. Cancer Res. 66, 3034-3043.

Ayala, I., Baldassarre, M., Giacchetti, G., Caldieri, G., Tete, S., Luini, A. and Buccione, R. (2008). Multiple regulatory inputs converge on cortactin to control invadopodia biogenesis and extracellular matrix degradation. J. Cell Sci. 121, 369-378.

Baldassarre, M., Pompeo, A., Beznoussenko, G., Castaldi, C., Cortellino, S., McNiven, M. A., Luini, A. and Buccione, R. (2003). Dynamin participates in focal extracellular matrix degradation by invasive cells. Mol. Biol. Cell 14, 1074-1084

Bougneres, L., Girardin, S. E., Weed, S. A., Karginov, A. V., Olivo-Marin, J. C., Parsons, J. T., Sansonetti, P. J. and Van Nhieu, G. T. (2004). Cortactin and Crk cooperate to trigger actin polymerization during Shigella invasion of epithelial cells. $J$. Cell Biol. 166, 225-235.

Bowden, E. T., Barth, M., Thomas, D., Glazer, R. I. and Mueller, S. C. (1999). An invasion-related complex of cortactin, paxillin and PKCmu associates with invadopodia at sites of extracellular matrix degradation. Oncogene 18, 4440-4449.

Bowden, E. T., Onikoyi, E., Slack, R., Myoui, A., Yoneda, T., Yamada, K. M. and Mueller, S. C. (2006). Co-localization of cortactin and phosphotyrosine identifies active invadopodia in human breast cancer cells. Exp. Cell Res. 312, 1240-1253.

Boyle, S. N., Michaud, G. A., Schweitzer, B., Predki, P. F. and Koleske, A. J. (2007). A critical role for cortactin phosphorylation by Abl-family kinases in PDGF-induced dorsal-wave formation. Curr. Biol. 17, 445-451.

Brabek, J., Constancio, S. S., Shin, N. Y., Pozzi, A., Weaver, A. M. and Hanks, S. K. (2004). CAS promotes invasiveness of Src-transformed cells. Oncogene 23, 7406-7415.

Brunton, V. G. and Frame, M. C. (2008). Src and focal adhesion kinase as therapeutic targets in cancer. Curr. Opin. Pharmacol. 8, 427-432.

Bruzzaniti, A., Neff, L., Sanjay, A., Horne, W. C., De Camilli, P. and Baron, R. (2005). Dynamin forms a Src kinase-sensitive complex with $\mathrm{Cbl}$ and regulates podosomes and osteoclast activity. Mol. Biol. Cell 16, 3301-3313.

Bruzzaniti, A., Neff, L., Sandoval, A., Du, L., Horne, W. C. and Baron, R. (2009). Dynamin reduces Pyk2 Y402 phosphorylation and SRC binding in osteoclasts. Mol. Cell. Biol. 29, 3644-3656. 
Buschman, M. D., Bromann, P. A., Cejudo-Martin, P., Wen, F., Pass, I. and Courtneidge, S. A. (2009). The novel adaptor protein Tks4 (SH3PXD2B) is required for functional podosome formation. Mol. Biol. Cell 20, 1302-1311.

Caldieri, G., Giacchetti, G., Beznoussenko, G., Attanasio, F., Ayala, I. and Buccione, R. (2008). Invadopodia biogenesis is regulated by caveolin-mediated modulation of membrane cholesterol levels. J. Cell Mol. Med. 13, 1728-1740.

Chen, W. T. (1989). Proteolytic activity of specialized surface protrusions formed at rosette contact sites of transformed cells. J. Exp. Zool. 251, 167-185.

Clark, E. S. and Weaver, A. M. (2008). A new role for cortactin in invadopodia: regulation of protease secretion. Eur. J. Cell Biol. 87, 581-590.

Clark, E. S., Whigham, A. S., Yarbrough, W. G. and Weaver, A. M. (2007). Cortactin is an essential regulator of matrix metalloproteinase secretion and extracellular matrix degradation in invadopodia. Cancer Res. 67, 4227-4235.

Cortesio, C. L., Chan, K. T., Perrin, B. J., Burton, N. O., Zhang, S., Zhang, Z. Y. and Huttenlocher, A. (2008). Calpain 2 and PTP1B function in a novel pathway with Src to regulate invadopodia dynamics and breast cancer cell invasion. J. Cell Biol. 180, 957-971.

Desmarais, V., Yamaguchi, H., Oser, M., Soon, L., Mouneimne, G., Sarmiento, C., Eddy, R. and Condeelis, J. (2009). N-WASP and cortactin are involved in invadopodium-dependent chemotaxis to EGF in breast tumor cells. Cell Motil. Cytoskeleton 66, 303-316.

Destaing, O., Sanjay, A., Itzstein, C., Horne, W. C., Toomre, D., De Camilli, P. and Baron, R. (2008). The tyrosine kinase activity of c-Src regulates actin dynamics and organization of podosomes in osteoclasts. Mol. Biol. Cell 19, 394-404.

Dierck, K., Machida, K., Mayer, B. J. and Nollau, P. (2009). Profiling the tyrosine phosphorylation state using SH2 domains. Methods Mol. Biol. 527, 131-155.

El Sayegh, T. Y., Arora, P. D., Fan, L., Laschinger, C. A., Greer, P. A., McCulloch, C. A. and Kapus, A. (2005). Phosphorylation of N-cadherin-associated cortactin by Fer kinase regulates N-cadherin mobility and intercellular adhesion strength. Mol. Biol. Cell 16, 5514-5527.

Gatesman, A., Walker, V. G., Baisden, J. M., Weed, S. A. and Flynn, D. C. (2004). Protein kinase Calpha activates c-Src and induces podosome formation via AFAP-110. Mol. Cell. Biol. 24, 7578-7597.

Guarino, M. (2010). Src signaling in cancer invasion. J. Cell Physiol. 223, 14-26.

Head, J. A., Jiang, D., Li, M., Zorn, L. J., Schaefer, E. M., Parsons, J. T. and Weed, S. A. (2003). Cortactin tyrosine phosphorylation requires Rac1 activity and association with the cortical actin cytoskeleton. Mol. Biol. Cell 14, 3216-3229.

Huang, C., Liu, J., Haudenschild, C. C. and Zhan, X. (1998). The role of tyrosine phosphorylation of cortactin in the locomotion of endothelial cells. J. Biol. Chem. 273, 25770-25776.

Linder, S. (2009). Invadosomes at a glance. J. Cell Sci. 122, 3009-3013.

Luxenburg, C., Parsons, J. T., Addadi, L. and Geiger, B. (2006). Involvement of the Src-cortactin pathway in podosome formation and turnover during polarization of cultured osteoclasts. J. Cell Sci. 119, 4878-4888.

Machida, K., Thompson, C. M., Dierck, K., Jablonowski, K., Karkkainen, S., Liu, B., Zhang, H., Nash, P. D., Newman, D. K., Nollau, P. et al. (2007). High-throughput phosphotyrosine profiling using SH2 domains. Mol. Cell 26, 899-915.

Nakahara, H., Mueller, S. C., Nomizu, M., Yamada, Y., Yeh, Y. and Chen, W. T. (1998). Activation of betal integrin signaling stimulates tyrosine phosphorylation of p190RhoGAP and membrane-protrusive activities at invadopodia. J. Biol. Chem. 273, 9-12.

Nyalendo, C., Michaud, M., Beaulieu, E., Roghi, C., Murphy, G., Gingras, D. and Beliveau, R. (2007). Src-dependent phosphorylation of membrane type I matrix metalloproteinase on cytoplasmic tyrosine 573, role in endothelial and tumor cell migration. J. Biol. Chem. 282, 15690-15699.

Nyalendo, C., Beaulieu, E., Sartelet, H., Michaud, M., Fontaine, N., Gingras, D. and Beliveau, R. (2008). Impaired tyrosine phosphorylation of membrane type 1-matrix metalloproteinase reduces tumor cell proliferation in three-dimensional matrices and abrogates tumor growth in mice. Carcinogenesis 29, 1655-1664.

Oikawa, T., Itoh, T. and Takenawa, T. (2008). Sequential signals toward podosome formation in NIH-src cells. J. Cell Biol. 182, 157-169.

Okada, M., Nada, S., Yamanashi, Y., Yamamoto, T. and Nakagawa, H. (1991). CSK: a protein-tyrosine kinase involved in regulation of src family kinases. J. Biol. Chem. 266, 24249-24252.

Onodera, Y., Hashimoto, S., Hashimoto, A., Morishige, M., Mazaki, Y., Yamada, A., Ogawa, E., Adachi, M., Sakurai, T., Manabe, T. et al. (2005). Expression of AMAP1, an ArfGAP, provides novel targets to inhibit breast cancer invasive activities. EMBO J. 24, 963-973.

Oser, M., Yamaguchi, H., Mader, C. C., Bravo-Cordero, J. J., Arias, M., Chen, X., Desmarais, V., van Rheenen, J., Koleske, A. J. and Condeelis, J. (2009). Cortactin regulates cofilin and N-WASp activities to control the stages of invadopodium assembly and maturation. J. Cell Biol. 186, 571-587.

Pichot, C. S., Hartig, S. M., Xia, L., Arvanitis, C., Monisvais, D., Lee, F. Y., Frost, J. A. and Corey, S. J. (2009). Dasatinib synergizes with doxorubicin to block growth, migration, and invasion of breast cancer cells. Br. J. Cancer 101, 38-47.

Poincloux, R., Lizarraga, F. and Chavrier, P. (2009). Matrix invasion by tumour cells: a focus on MT1-MMP trafficking to invadopodia. J. Cell Sci. 122, 3015-3024.

Rothschild, B. L., Shim, A. H., Ammer, A. G., Kelley, L. C., Irby, K. B., Head, J. A., Chen, L., Varella-Garcia, M., Sacks, P. G., Frederick, B. et al. (2006). Cortactin overexpression regulates actin-related protein $2 / 3$ complex activity, motility, and invasion in carcinomas with chromosome 11q13 amplification. Cancer Res. 66, 8017-8025.

Sakurai-Yageta, M., Recchi, C., Le Dez, G., Sibarita, J. B., Daviet, L., Camonis, J., D'Souza-Schorey, C. and Chavrier, P. (2008). The interaction of IQGAP1 with the exocyst complex is required for tumor cell invasion downstream of Cdc42 and RhoA. J. Cell Biol. 181, 985-998.

Sandilands, E., Cans, C., Fincham, V. J., Brunton, V. G., Mellor, H., Prendergast, G. C., Norman, J. C., Superti-Furga, G. and Frame, M. C. (2004). RhoB and actin polymerization coordinate $\mathrm{Src}$ activation with endosome-mediated delivery to the membrane. Dev. Cell 7, 855-869.

Seals, D. F., Azucena, E. F., Jr, Pass, I., Tesfay, L., Gordon, R., Woodrow, M., Resau, J. H. and Courtneidge, S. A. (2005). The adaptor protein Tks $5 /$ Fish is required for podosome formation and function, and for the protease-driven invasion of cancer cells. Cancer Cell 7, 155-165.

Singh, A., Sowjanya, A. P. and Ramakrishna, G. (2005). The wild-type Ras: road ahead. FASEB J. 19, 161-169.

Spinardi, L., Rietdorf, J., Nitsch, L., Bono, M., Tacchetti, C., Way, M. and Marchisio, P. C. (2004). A dynamic podosome-like structure of epithelial cells. Exp. Cell Res. 295, 360-374.

Stuible, M., Dube, N. and Tremblay, M. L. (2008). PTP1B regulates cortactin tyrosine phosphorylation by targeting Tyr446. J. Biol. Chem. 283, 15740-15746.

Stylli, S. S., Stacey, T. T., Verhagen, A. M., Xu, S. S., Pass, I., Courtneidge, S. A. and Lock, P. (2009). Nck adaptor proteins link Tks5 to invadopodia actin regulation and ECM degradation. J. Cell Sci. 122, 2727-2740.

Summy, J. M. and Gallick, G. E. (2003). Src family kinases in tumor progression and metastasis. Cancer Metastasis Rev. 22, 337-358.

Tehrani, S., Tomasevic, N., Weed, S., Sakowicz, R. and Cooper, J. A. (2007). Src phosphorylation of cortactin enhances actin assembly. Proc. Natl. Acad. Sci. USA 104, 11933-11938.

Thomas, S. M. and Brugge, J. S. (1997). Cellular functions regulated by Src family kinases. Annu. Rev. Cell Dev. Biol. 13, 513-609.

Walker, V. G., Ammer, A., Cao, Z., Clump, A. C., Jiang, B. H., Kelley, L. C., Weed, S. A., Zot, H. and Flynn, D. C. (2007). PI3K activation is required for PMA-directed activation of cSrc by AFAP-110. Am. J. Physiol. Cell Physiol. 293, C119-C132.

Webb, B. A., Eves, R. and Mak, A. S. (2006). Cortactin regulates podosome formation: roles of the protein interaction domains. Exp. Cell Res. 312, 760-769.

Webb, B. A., Jia, L., Eves, R. and Mak, A. S. (2007). Dissecting the functional domain requirements of cortactin in invadopodia formation. Eur. J. Cell Biol. 86, 189-206.

Xue, C., Wyckoff, J., Liang, F., Sidani, M., Violini, S., Tsai, K. L., Zhang, Z. Y., Sahai, E., Condeelis, J. and Segall, J. E. (2006). Epidermal growth factor receptor overexpression results in increased tumor cell motility in vivo coordinately with enhanced intravasation and metastasis. Cancer Res. 66, 192-197.

Yamaguchi, H. and Condeelis, J. (2007). Regulation of the actin cytoskeleton in cancer cell migration and invasion. Biochim. Biophys. Acta 1773, 642-652.

Yamaguchi, H., Lorenz, M., Kempiak, S., Sarmiento, C., Coniglio, S., Symons, M., Segall, J., Eddy, R., Miki, H., Takenawa, T. et al. (2005). Molecular mechanisms of invadopodium formation: the role of the N-WASP-Arp $2 / 3$ complex pathway and cofilin. J. Cell Biol. 168, 441-452.

Yamaguchi, H., Takeo, Y., Yoshida, S., Kouchi, Z., Nakamura, Y. and Fukami, K. (2009). Lipid rafts and caveolin-1 are required for invadopodia formation and extracellular matrix degradation by human breast cancer cells. Cancer Res. 69, 85948602.

Yeatman, T. J. (2004). A renaissance for SRC. Nat. Rev. Cancer 4, 470-480. 